\title{
SACROCOCCYGEAL PILONIDAL DISEASE: ANALYSIS OF PREVIOUSLY PROPOSED RISK FACTORS
}

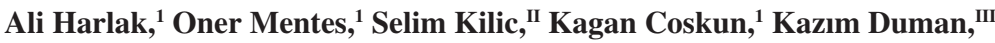 \\ Fahri Yilmaz ${ }^{\mathrm{IV}}$
}

doi: $10.1590 / \mathrm{S1807-59322010000200002}$

Harlak A, Mentes O, Kilic S, Duman K, Yilmaz F. Sacrococcygeal pilonidal disease: analysis of previously proposed risk factors. Clinics. 2010;65(2):125-31.

PURPOSE: Sacrococcygeal pilonidal disease is a source of one of the most common surgical problems among young adults. While male gender, obesity, occupations requiring sitting, deep natal clefts, excessive body hair, poor body hygiene and excessive sweating are described as the main risk factors for this disease, most of these need to be verified with a clinical trial. The present study aimed to evaluate the value and effect of these factors on pilonidal disease.

METHOD: Previously proposed main risk factors were evaluated in a prospective case control study that included 587 patients with pilonidal disease and 2,780 healthy control patients.

RESULTS: Stiffness of body hair, number of baths and time spent seated per day were the three most predictive risk factors. Adjusted odds ratios were 9.23, 6.33 and 4.03, respectively $(\mathrm{p}<0.001)$. With an adjusted odds ratio of 1.3 (p<.001), body mass index was another risk factor. Family history was not statistically different between the groups and there was no specific occupation associated with the disease.

CONCLUSIONS: Hairy people who sit down for more than six hours a day and those who take a bath two or less times per week are at a 219-fold increased risk for sacrococcygeal pilonidal disease than those without these risk factors. For people with a great deal of hair, there is a greater need for them to clean their intergluteal sulcus. People who engage in work that requires sitting in a seat for long periods of time should choose more comfortable seats and should also try to stand whenever possible.

KEYWORDS: Pilonidal disease; Pilonidal sinus; Sacrococcygeal; Risk factors; Etiology; Stiffness of body hair; Body Mass Index; BMI.

\section{INTRODUCTION}

Sacrococcygeal pilonidal disease (SPD) is a well recognized source of a common surgical problem affecting primarily white men between puberty and their early thirties. ${ }^{1}$ The name of the disease originated from Latin,

\footnotetext{
${ }^{\mathrm{I}}$ Departmen of General Surgery, Gulhane Military Medical Academy Ankara, Turkey

II Department of Public Health, Gulhane Military Medical Academy - Ankara, Turkey

III Department of General Surgery, Gumussuyu Military Hospital - Istanbul, Turkey

${ }^{\text {IV }}$ Department of General Surgery, Golcuk Military Hospital - Golcuk, Turkey Email: onermentes@yahoo.com

Tel.: 903123045015

Received for publication on August 17, 2009

Accepted for publication on October 26, 2009
}

meaning nest of hair. The disease frequently occurs as a chronic infection of the skin in the region of the buttock crease. SPD was first described by Hodges in 1880 and later, in 1833, was described by Herbert Mayo as a hair-containing sinus. ${ }^{2}$ Mayo's findings were of a characteristic epithelial track (the sinus) that generally contains hair and is located in the skin of the natal cleft. The condition was commonly diagnosed in jeep drivers during the Second World War, which led to it being known as "jeep disease."” Although not as common, pilonidal disease may occur in other body parts, such as the clefts between the fingers of barbers or hairdressers. ${ }^{4}$

For a long time, the cause of SPD has been a matter of debate. Until the second half of the $20^{\text {th }}$ century, SPD was thought to be secondary to a congenital remnant of an epithelial-lined tract of postcoccygeal epidermal cell 
rests or vestigial scent cells. A variety of explanations have supported the congenital theory. Some authors have suggested that the persistence of a caudal remnant of the neural canal forms small cysts, which cause SPD. ${ }^{5}$ Another hypothesis for SPD provides that SPD is the result of a formation of dermal inclusions by the cystic changes of sequestered epithelial rests. ${ }^{6}$ Excessive involution of the human tail has been accused of attracting skin into a subcutaneous area and results in an epithelium-lined tract. ${ }^{7}$ SPD is now, however, widely accepted as an acquired disorder. SPD is more common in men and in hirsute people. ${ }^{1}$ It usually occurs after puberty, and a linkage between the disease and various occupations has been demonstrated. ${ }^{1}$ In addition, there is a high recurrence rate of SPD after surgical excision. These factors argue against the validity of the congenital theory to explain SPD. Further, congenital tracts do not contain hair and are lined by cuboidal epithelium. ${ }^{8}$

Male gender, obesity, occupations or sports requiring sitting, a deep natal cleft, excessive body hair, stiff or coarse hair, poor body hygiene and excessive sweating are described as the primary risk factors for SPD. ${ }^{9}$ However, there is a noted lack of prospective studies in the literature that analyze and confirm these risk factors. There are only a few studies evaluating risk factors for SPD, and they are generally focused on the role of obesity. ${ }^{11,12}$

The present study investigated the previously described risk factors. Body mass index (BMI), profession, time spent seated per day, stiffness of body hair, number of baths per week and family history were explored in a large-scale prospective case control trial. The purpose of the current study was to determine the clinical value and quantitative effect of significant risk factors on the pathogenesis of SPD. The results will help to form evidence-based advice for the prevention of the disease. The findings may be useful in correlating the proposed etiological theory with clinical practice and may also be a starting point for minimally invasive or non invasive treatment options.

\section{MATERIAL AND METHOD}

Study design: A total of 587 patients with SPD and 2,780 healthy control patients were enrolled for the study. All data were collected prospectively during a 3-year time period and the study was designed as a case-control trial.

Patient Selection: Patients in active military service who were diagnosed with SPD in five military hospitals during the study period were enrolled in the trial. The two inclusion criteria included being a soldier in active military service and the presence of SPD. Regardless of previous or further treatment of the disease or co-morbid conditions, all patients with SPD who consented to participate in the study were subjected to a physical examination and clinical interview.

Selection of control patients: The control patients were selected from soldiers who presented for their first medical examination when they were joining the army. All soldiers underwent a first medical evaluation as a part of the standard procedure; however, the soldiers in the control group were examined more carefully for the study parameters. Patients with current or previous, symptomatic or asymptomatic SPD and soldiers who did not consent to study participation were excluded from the control group.

Data Collection: With the aim of evaluating previously defined risk factors, a data collection form was designed (Table 1). All physicians who would work in data collection were informed about the description of the data. Surgeons filled in the data collection forms during the first meeting with the patients. For the control group, physicians examined and filled in the data collection form at a recruit's initial medical exam.

During these examinations, demographic and basic medical data, including patients' profession, age, sex, weight and height were documented. Moreover, time spent seated per day, body hair rate, number of baths per week and family history of SPD were recorded by the physicians for both patients with SPD and control patients.

Patient history and physical examination findings in the patient group were recorded for routine clinical evaluation; such data were used for another descriptive paper that discusses the clinical presentation of disease.

Outcome Parameter: Age, sex, BMI, profession, time spent seated per day, body hair rate, number of baths per week and family history were compared between the

Table 1 - Data collection form

\section{Name:}

Patient ( ), Control ( )

Date and hospital name:

Age and sex:

Length $(\mathrm{cm})$ :

Weight (kg):

Profession ${ }^{(*)}$

Time spent seated per day: $<6$ hour ( ), $\geq 6$ hour ( )

Body hair rate: hairless ( ), mildly hairy ( ), hairy ( )

Number of baths in a week:

Family history ${ }^{(* *)}$ Positive ( ), Negative ( )

\footnotetext{
${ }^{(*)}$ The job mostly done during the two years prior to the sacrococcygeal pilonidal disease (SPD). If the patient had worked, but not in the same job for more than six months during this time period, they were considered to be an irregular worker.

${ }^{(* *)}$ Presence of SPD in father, mother, brother or sister.
} 
patients with SPD and the control patients. The odds ratio (OR) for every parameter and for the three most predictive risk factors were calculated. The OR for all predictive risk factors were adjusted with a multivariate analysis for age, BMI, time spent seated per day, body hair rate and number of baths per week.

\section{Description of data}

BMI: Weight (kilogram) and height (meter) for each patient and control were measured and BMI was calculated by the standard formula (weight / height ${ }^{2}$ ). Case and controls were divided into three groups, which are as follows: normal (BMI<25), overweight (BMI between 25-30) and obese (BMI >30).

Occupation: The occupation performed the most during two years prior to the diagnosis of SPD was accepted as the patient's profession. If the patient had worked, but not in the same occupation for more than six months during this time period, the patient was considered an irregular worker.

Time spent seated per day: The physician asked each patient about the time period that they spent sitting on a seat during their usual work practice. Patients were then grouped into two groups including those who spent more and those who spent less than six hours of sitting time. "Six hours" is accepted as the maximum possible sitting time of a worker who has a job requiring sitting down for prolonged period.

Body hair rate: Body hair rate was also graded subjectively. The levels used for the study are as follows: "hairless" if the intergluteal sulcus was clean with a minimal amount of hair, "hairy" if the intergluteal sulcus was full of hair and "mild hairy" if there were few hairs.

Number of baths in a week: The bath and shower habits of the patients and controls were determined by interview and the approximate number of baths per week was recorded.

Family history about SPD: Presence of SPD in one or more family members (i.e., father, mother, brother or sister) was self-reported by the patients and subsequently recorded by interviewers.

Statistical Analysis: Statistical analysis was performed with SPSS software version 11.5 (SPSS Inc, Chi., II., USA). Clinical data were expressed as means \pm standard deviation, ranges and percentages. We investigated the effects of the variables on SPD by calculating crude (unadjusted) odds ratios in univariate logistic regression analyses. We then conducted multivariate analyses. We reduced the model by using a backward elimination method and eliminated potential risk factors by using likelihood ratio tests. A p-value of $<0.05$ was accepted as statistically significant.

\section{RESULTS}

During the study period, 587 patients and 2,780 controls were evaluated.

Age and Sex: All patients and controls were white and male. The mean age was $22.49 \pm 2.97$ in the patient group, as compared with $21.02 \pm 1.73$ for the control group. The crude OR and the adjusted OR were 1.32 and 1.31, respectively. The $95 \%$ confidence interval (CI) was $1.27-1.38$ and 1.251.37 , respectively.

BMI: Mean BMI was slightly higher in patients with SPD. It was $23.8 \pm 2.7$ (range 16.1-36.8) in the patient group and $22.7 \pm 2.5$ (range 16.1-36.9) in the control group. $(\mathrm{p}<0.001)$. The majority of patients $(70.5 \%)$ and controls $(83.5 \%)$ had a BMI under 25 (Table 3). Individuals having a BMI less than 25 were used as the reference. Based on the comparison of the normal weight and overweight groups, the crude OR was 2.14 (95\% CI: 1.74-2.64) and the adjusted OR was 1.56 (95\% CI: 1.20-2.03). The difference was statistically significant in both univariate and multivariate analysis $(\mathrm{p}<0.001$ and $\mathrm{p}=0.001$, respectively). Only $2 \%$ of patients and $1.3 \%$ of controls were in the obese group and the difference in OR was not significant in the univariate and multivariate analyses when compared with normal weight individuals. ( $\mathrm{p}=0.08$ and $\mathrm{p}=0.598$ respectively) (Table 2 ).

Occupation: A total of 56 job titles were described for both groups. The most commonly reported occupations in the patient group were farmers $(15 \%)$, irregular workers (13.3\%), Salespeople (11.9\%), unemployed (11.4\%) and drivers $(5.8 \%)$. The most popular jobs in the control group were irregular workers $(20.4 \%)$, unemployed $(12.1 \%)$, drivers $(6.7 \%)$, farmers $(6.04 \%)$ and technicians $(5.4 \%)$. There was no particular occupation associated with SPD.

Time Spent Seated Per Day: Time spent seated per day was significantly higher in the patient group. A total of 56\% of patients sat down on a seat for more than six hours, as compared with $27.8 \%$ of controls $(\mathrm{p}<0.001)$ (Table 3$)$. The crude OR was $3.31(95 \% \mathrm{CI}=2.75-3.97)$ and the adjusted OR was 4.03 (95\% CI= 3.22- 5.05) for individuals who sat down for more than six hours, as compared with the group who sat down for less than six hours per day $(\mathrm{p}<0.001)$ (Table 2).

Body Hair Rate: The percentage of hairy and mildly hairy persons was significantly higher in the patient group than in the control group (39.5\% and $52.5 \%$, as compared with $14.0 \%$ and $41.8 \%$, respectively) $(\mathrm{p}<0.001)$ (Table $3)$. The crude OR was calculated as 6.93 for mildly hairy people (95\% CI=5.05-9.52) and 15.54 for hairy people (95\% CI=11.13-21.70). The adjusted OR was also highly significant for both groups. The adjusted OR was $5.15(95 \%$ CI: 3.65-7.27) for the mildly hairy group and 9.23 (95\% CI: 
Table 2- Effects of various variables on pilonodial disease based on univariate statistics and multivariate logistic regression analysis

\begin{tabular}{|c|c|c|c|c|c|c|}
\hline & Unadjusted OR & $95 \% \mathrm{CI}$ & $\mathrm{P}$-value & Adjusted $^{(\mathrm{a})} \mathrm{OR}$ & $95 \% \mathrm{CI}$ & P-value \\
\hline \multicolumn{7}{|l|}{ Body Mass Index } \\
\hline \multicolumn{7}{|c|}{$<25$ (Reference) } \\
\hline $25-30$ & 2.14 & $1.74-2.64$ & $<0.001$ & 1.56 & $1.20-2.03$ & 0.001 \\
\hline$>30$ & 1.82 & $0.94-3.52$ & 0.08 & 1.26 & $0.54-2.94$ & 0.60 \\
\hline \multicolumn{7}{|l|}{ Time Spent Seated Per Day } \\
\hline \multicolumn{7}{|c|}{$<6$ hour (Reference) } \\
\hline$\geq 6$ hour & 3.31 & $2.75-3.97$ & $<0.001$ & 4.03 & $3.22-5.05$ & $<0.001$ \\
\hline \multicolumn{7}{|l|}{ Body Hair Rate } \\
\hline \multicolumn{7}{|c|}{ Hairless (Reference) } \\
\hline Mild & 6.93 & $5.05-9.52$ & $<0.001$ & 5.15 & $3.65-7.27$ & $<0.001$ \\
\hline Hairy & 15.54 & $11.13-1.70$ & $<0.001$ & 9.23 & $6.31-13.51$ & $<0.001$ \\
\hline \multicolumn{7}{|l|}{ Number of Baths in a Week } \\
\hline \multicolumn{7}{|c|}{3 or more (Reference) } \\
\hline 2 or less & 5.50 & $4.52-6.70$ & $<0.001$ & 6.33 & $5.02-7.99$ & $<0.001$ \\
\hline
\end{tabular}

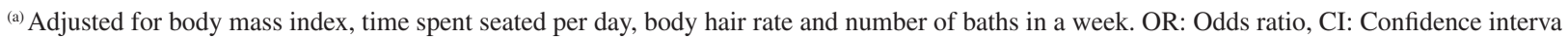

Table 3- Distribution of risk factors in the patient and control groups

\begin{tabular}{|c|c|c|c|c|c|c|c|}
\hline & \multicolumn{2}{|c|}{ Patient } & \multicolumn{2}{|c|}{ Control } & \multicolumn{2}{|c|}{ Total } & \multirow[t]{2}{*}{ P-value } \\
\hline & $\mathrm{n}$ & $\%$ & $\mathrm{n}$ & $\%$ & $\mathrm{n}$ & $\%$ & \\
\hline \multicolumn{8}{|l|}{ Body Mass Index } \\
\hline$<25$ & 414 & 70,5 & 2321 & 83.5 & 2735 & 81.2 & \\
\hline $25-30$ & 161 & 27.4 & 422 & 15.2 & 583 & 17.3 & 0.001 \\
\hline$>30$ & 12 & 2.0 & 37 & 1.3 & 49 & 1.5 & 0.60 \\
\hline \multicolumn{8}{|l|}{ Time sitting on a seat per day } \\
\hline$<6$ hour & 258 & 44.0 & 2006 & 72.2 & 2264 & 67.2 & \\
\hline$\geq 6$ hour & 329 & 56.0 & 774 & 27.8 & 1103 & 32.8 & $<0.001$ \\
\hline \multicolumn{8}{|l|}{ Stiffness of Body Hair } \\
\hline Hairless & 47 & 8.0 & 1228 & 44.2 & 1275 & 37.9 & \\
\hline Mild & 308 & 52.5 & 1161 & 41.8 & 1469 & 43.06 & $<0.001$ \\
\hline Hairy & 232 & 39.5 & 390 & 14.0 & 622 & 18.5 & $<0.001$ \\
\hline \multicolumn{8}{|l|}{ Number of Baths in a Week } \\
\hline 3 or more & 168 & 28.62 & 1913 & 68.8 & 2081 & 61.8 & \\
\hline 2 or less & 419 & 71.38 & 867 & 31.2 & 1286 & 38.2 & $<0.001$ \\
\hline \multicolumn{8}{|c|}{ Family History for Sacrococcygeal Pilonidal Disease (SPD) } \\
\hline Positive & 98 & 16.7 & 405 & 14.6 & 503 & 14.9 & \\
\hline Negative & 489 & 83.3 & 2375 & 85.4 & 2864 & 85.1 & \\
\hline
\end{tabular}

6.31-13.51) for the hairy group. The p-value was $<0.001$ for all analyses concerning body hair rate (Table 2).

Number of Baths in a Week: The mean number of baths in a week was $2.26 \pm 0.57$ in the patient group and $3.04 \pm 1.17$ in the control group $(\mathrm{p}<0.001)$. The rate of people who take a bath two times or less per week was
$71.4 \%$ in the patient group and $31.2 \%$ in the control group (Table 3). The crude OR for SPD was 5.50 -fold $(95 \%$ CI:4.52-6.70) and adjusted OR was 6.33-fold (95\% CI:5.027.99) higher in people who take two or less showers or baths per week compared with those who take three or more baths per week $(\mathrm{p}<0.001)($ Table 2$)$. 
Family History of SPD: Family history of SPD was slightly higher in the patient group, but it was not statistically significant. Presence of SPD in first degree family members was exposed in the family histories of 98 patients $(16.7 \%)$ and 405 controls $(14.6 \%)(\mathrm{p}=0.189)$ (Table 3).

The three most predictive risk factors were body hair rate, bathing habituation and sitting time. A total of 111 people from both the patient and control groups possessed these three risk factors simultaneously and 80 of those individuals were from patient group $(72.07 \%)$. In contrast, these 3 risk factors were all absent in 602 people, and only 7 of these were from patient group (1.16\%). With a logistic regression analysis, the risk is 219 -fold higher for hairy people who shower or bath two or less times per week and sit more than six hours per day compared with hairless people who shower or bath three or more times per week and sit less than six hours in a day, where OR is 219.36 and $95 \%$ $\mathrm{CI}$ is $93.50-514.62(\mathrm{p}<0.001)$.

\section{DISCUSSION}

Studies based on large patient series about SPD are usually reported from army hospitals. ${ }^{12-14}$ The patient records analyzed in this study revealed that SPD is the second commonly seen surgical pathology among soldiers. Patient number and homogeneity of groups provide researchers with an opportunity to design prospective, large-scale clinical trials to evaluate risk factors for SPD. While soldiers are usually young active males who are not representative of general population, pilonidal disease affects mainly white males in this age group and study results may be applied to a broad population.

From its first description in 1930 to the middle of $20^{\text {th }}$ century, many reaearchers have attempted to explain SPD with a congenital etiology based theory. Patey and Scarff first proposed the hypothesis that the disease results from suction of hair from the surrounding skin..$^{15}$ They maintain that the pilonidal cyst is a chronic disease caused by penetration of short, stiff hairs into the subcutaneous tissues and create of foreign body reaction. Bascom showed that the midline pits are enlarged and distorted hair follicles. ${ }^{16}$ Enlarged hair follicles in the midline are the portals of entry for the hairs and extension occurs by persistent low-grade infection. ${ }^{8}$ Gravity and motion of the gluteal folds have been suggested as a cause of creation of a vacuum that pulls on the follicles. ${ }^{17}$ To explain how these hairs make their way into the tissues, Karydakis attributed the cause to three main factors. ${ }^{14}$ Those factors include the invader, which is the loose hair; the force, which causes the insertion; and the vulnerability of the skin to the insertion of hair at the depth of the natal cleft. Previously described risk factors are acting on these causation factors.

Hair is the main initiative agent in all theories that attempt to explain the pathogenesis of SPD, which may be the reason for the male predomination of the disease. Hair is the invader agent according to Karydakis theory. ${ }^{14}$ Clinical observations also revealed that patients with SPD are usually hairy men. The present study evaluated body hair rate as a risk factor. Increasing body hair rate was matched to 9.23-fold higher risk of SPD even after adjustment for other risk factors. The graduation of hair rate was made by physicians according to a three-graded subjective scale. Using this scale, it is relatively easy to distinguish hairy and hairless people. A mild hairy group is defined for possible confusions. Risk for SPD was significantly higher for the hairy and mild hairy groups. An objective measurement technique may be used for definition of body hair rate. This technique will help to obtain objective results, however, it must be simple to use in a large patient group.

While there is no previous study evaluating the relation between the bathing habituation and SPD, we hypothesized that this relation affected the amount of loose hair at the intergluteal groove. The adjusted risk of SPD diagnosis is 6.33-fold greater for those who bath two or less times per week than the risk for those people who take three or more baths per week. The results revealed that the cleaning of intergluteal sulcus may prevent this disease. The definition of bathing number is relatively subjective and determined by patient self-reporting. A prospective randomized trial aimed to detect effect of bathing habituation is not easy. A very large sample size and long follow-up time are needed to conduct such a study.

SPD is rare with black and Asian people. ${ }^{89}$ Turkish people are mostly white and the number of black people is negligible. All of the individuals in the patient and control groups were white males in this study.

SPD was referred to as the "jeep disease" during Second World War. ${ }^{3}$ Drivers are known as an at risk group for this condition. Further articles mentioned to the relationship of occupation and SPD. Several case reports have described a similar condition arising in the clefts between the fingers of barbers or hairdressers. ${ }^{18}$ We recorded and analyzed occupation in both the patient and control groups, but could not determine any profession indicating SPD. Some limitations have made a systematic analysis difficult to achieve; for example, it is difficult to classify occupations into meaningful groups. Although we tried to evaluate occupations, a total of 56 job titles were used and most of the individuals in the patient and control groups did not have a regular job. It is possible to say that there were more drivers in the control group compared with patient group. 
Current cars and trucks are comfortable and roads are flat. It is possible that improved vehicle and road conditions are the reason today's drivers experience SPD less often than with their colleagues who rode in a jumping jeep on bumpy field roads during the Second World War. It is not easy to deny the relation between occupation and SPD according to study results; although occupation seems to be indirectly related to sitting time and body cleaning.

Sitting time in a day on a seat was another risk factor evaluated in the study. The adjusted risk of SPD was 4.3fold higher for individuals who were sitting more than six hours in a day. The majority of the classical articles about SPD indicate a relation between the long sitting time and this disease; however, we could not find a clinical trial in the literature that was showing this relation.

The role of obesity as a causative factor and its effect on the effectiveness of some treatment modalities has been discussed in the literature. In a clinical trial containing 419 patients and 213 controls, BMI was reported to be slightly higher in the patient group, but the difference was not statistically significant. ${ }^{10}$ In another study, the authors declared that the Karydakis procedure for managing chronic pilonidal sinus in obese patients was easy to perform, had a fast healing time, required a short hospital stay, enabled a rapid return to work and resulted in a low recurrence rate. ${ }^{19}$ In contrast, Cubukcu et all. stated that obese patients with high BMI have a higher risk of recurrence of pilonidal sinus disease after surgical intervention. ${ }^{11} \mathrm{~A}$ recent paper that included 125 patients and 125 controls revealed that sacrococcygeal subcutaneous fatty tissue is thicker in the patient group. ${ }^{20} \mathrm{In}$ the present study, the independent risk was calculated as 1.56 and 1.26 for overweight and obese people, respectively, compared with people who had a BMI under 25. Most of the patients and all of the controls in this study were soldiers in active military service, and all were young people. Because there were only a few obese individuals in both groups, this group could not be analyzed; however, the overweight group was appropriate for analysis. The effect of BMI may be better evaluated in a community-based population. Our results revealed that obesity is a relatively less important risk factor for SPD. The intergluteal groove is a deep moist area in which broken hairs and foreign bodies can collect, leading to infection or invasion of the skin. The deepness of the intergluteal sulcus may be different in people based on BMI or personal body characteristics. We thought that the depth of the intergluteal sulcus can affect the amount of loose hair collected in it.

The prevalence of a family history of SPD was around $15 \%$ in both groups, a difference that was not statistically significant. These findings suggest that this condition does not occur on a congenital basis.

The mean age was $22.49 \pm 2.97$ in the patient group, as compared with $21.02 \pm 1.73$ for the control group. Although the finding was not clinically significant due to the large sample size, the result was statistically significant $(\mathrm{p}<0.001)$.

\section{CONCLUSION}

Hirsute people who sit for longer periods of time and have a decreased number of baths per week have a 219-fold increased risk for SPD. This finding is extremely significant and important because simple precautions may be proposed for prevention. Careful cleaning of the intergluteal sulcus should be conducted, especially among people who are hirsute. Hairy people should take more baths and use skin care products that reduce unwanted hairs. Bathing facilities and opportunities to bath must be arranged for people who live communally, such as soldiers. People who have occupations requiring sitting down in a seat for long hours must choose more comfortable seats and must try to stand up on every occasion.

\section{REFERENCES}

1. Chintapatla S, Safarani N, Kumar S, Haboubi N. Sacrococcygeal pilonidal sinus: historical review, pathological insight and surgical options. Tech Coloproctol. 2003;7:3-8.

2. Hodge RM. Pilonidal sinus. Poston Med Surg J. 1880;103:485-6.

3. Classic articles in colonic and rectal surgery. Louis A. Buie, M.D. 18901975: Jeep disease (pilonidal disease of mechanized warfare). Dis Colon Rectum. 1982;25:384-90.

4. Patel MR, Bassini L, Nashad R, Anselmo MT. Barber's interdigital pilonidal sinus of the hand: a foreign body hair granuloma. J Hand Surg. 1990;15:652-5.
5. Gage M. Pilonidal sinus: an explanation of its embryologic development. Arch Surg. 1935;31:175-89.

6. Bookman MR. Treatment of the sacrococcygeal sinus (pilonidal sinus). N Y State J Med 1924;24:204.

7. Lannelongue O. Memoir sur les fistules et les depression cutannes congenitals inferieures. Observation d'un kyste dermoid de la region sacro-coccygene. Bull Soc Chir Paris. 1882;8:185-94.

8. da Silva JH. Pilonidal cyst: cause and treatment. Dis Colon Rectum. 2000;43:1146-56. 
9. Hull TL, Wu J. Pilonidal disease. Surg Clin North Am. 2002;82:1169-85.

10. Cubukçu A, Carkman S, Gönüllü NN, Alponat A, Kayabaşi B, Eyüboğlu E. Lack of evidence that obesity is a cause of pilonidal sinus disease. Eur J Surg. 2001;167:297-8.

11. Cubukçu A, Gönüllü NN, Paksoy M, Alponat A, Kuru M, Ozbay O. The role of obesity on the recurrence of pilonidal sinus disease in patients, who were treated by excision and Limberg flap transposition. Int $\mathbf{J}$ Colorectal Dis. 2000;15:173-5.

12. Mentes O, Bagci M, Bilgin T, Coskun I, Ozgul O, Ozdemir M. Management of pilonidal sinus disease with oblique excision and primary closure: results of 493 patients. Dis Colon Rectum. 2006;49:104-8.

13. Doll D, Friederichs J, Dettmann H, Boulesteix AL, Duesel W, Petersen $\mathrm{S}$. Time and rate of sinus formation in pilonidal sinus disease. Int $\mathbf{J}$ Colorectal Dis. 2008;23:359-64.

14. Karydakis GE. New approach to the problem of pilonidal sinus. Lancet 1973;2:1414-15.
15. Patey DH, Scarff RW. Pathology of postanal pilonidal sinus: its bearing on treatment. Lancet. 1946;2:484-6.

16. Bascom J. Pilonidal sinus: origin from follicles of hairs and results of follicle removal as treatment. Surgery. 1980;87:567-72.

17. Brearley R. Pilonidal sinus. A new theory of origin. Br J Surg. 1955;43:455-62.

18. Efthimiadis C, Kosmidis C, Anthimidis G, Grigoriou M, Levva $\mathrm{S}$, Fachantidis $\mathrm{P}$, et al. Barber's hair sinus in a female hairdresser: uncommon manifestation of an occupational disease: a case report. Cases J. 2008;1:214.

19. Sakr M, El-Hammadi H, Moussa M, Arafa S, Rasheed M. The effect of obesity on the results of Karydakis technique for the management of chronic pilonidal sinus. Int J Colorectal Dis. 2003;18:36-9.

20. Balik O, Balik AA, Polat KY, Aydinli B, Kantarci M, Aliagaoglu C, et al. The importance of local subcutaneous fat thickness in pilonidal disease. Dis Colon Rectum. 2006;49:1755-7. 